\title{
Power Density and SAR in Multi-Layered Life Tissue at Global System Mobile (GSM) Frequencies
}

\author{
Khitam El Wasife
}

Deptartment of Physics, College of Science, Islamic University, Gaza, Palestinian Territory.

Email: kelwasife@iugaza.edu.ps

Received June $12^{\text {th }}, 2011$; revised July $13^{\text {th }}, 2011$; accepted July $23^{\text {rd }}, 2011$.

\begin{abstract}
In this paper, the power density and specific absorption rate (SAR) distribution in multi-layered life tissues and exposed to electromagnetic field emitted from handheld cellular phone operating in the $900 \mathrm{MHz}$ and $1800 \mathrm{MHz}$ is studied. A One dimensional Finite-Difference Time-Domain (FDTD) method has been used, and numerical assessment of electromagnetic fields coupled to four biological body tissue. Values of interest in these assessments include induced electric field and specific absorption rate (SAR), which has been a measured of absorbed power density in the body have been evaluated. A comparison of the effect of two frequencies on human body tissue has shown graphically. The results noticed that the effect of frequency $900 \mathrm{MHz}$ by mobile phone Global System Mobile (GSM) is more than the frequency of $1800 \mathrm{MHz}$.
\end{abstract}

Keywords: Electromagnetic Waves, Life Tissues, Specific Absorption Rate, Power Density, Finite Difference Time Domain Method (FDTD)

\section{Introduction}

The use of mobile phone by people has grown substantially in the last years. In parallel with this, an increased concern by the scientific community, the authorities and the population regarding the safety of these phones has raised. It is currently the most appropriate choice when highly non-homogeneous structures are involved for which boundary techniques have fundamental limitations [1].

The Finite-Difference Time-Domain (FDTD) is the most often used method for evaluating of electromagnetic fields in human tissue.

The heating effects resulted from using a mobile phone operating near a metal wall has been studied [2]. The finite-difference time-domain (FDTD) simulation scheme was used in the simulation. The simulated physical domain consists of a dipole antenna, a high-resolution human head model.

The temperature rise in a human head caused by absorbed power in the form of SAR from a cellular telephone has been computed using a bio-heat equation approach. The SAR is computed using an FDTD solver for a reasonable cellular telephone model that radiates at both 900 and $1800 \mathrm{MHz}$ [3]. The issue of temperature rise in the eye caused by electromagnetic fields has generated some interest due to the low blood flow through the eye tissue has been evaluated [4].

Computation of electromagnetic field inside a tissue at mobile communication has been studied, which presents a new approach to calculate the electromagnetic field inside a tissue, composed of electrically excitable cell by means of the FDTD (finite difference time domain method) $[5,6]$.

A simple planar multi-layered head model irradiated by plane wave in the frequency range of $100 \mathrm{MHz}-300$ Ghz is investigated by Akram and Andrew in [7], and it consists of skin, fat, bone, Dura, CSF and brain. Modeling the effect of electromagnetic waves produced by mobile phone base station on human body tissue has been studied [8].

The electromagnetic waves of different power levels and different frequencies penetrate into the human body causing health risks; this is of great public concern.

In this paper, a simple planar multi-layered life tissue model will be used to calculate the electric field, magnetic field, power density and Specific Absorption Rate (SAR) distributions inside the model when exposed to an electromagnetic waves with different frequencies produc- 
ed from mobile phone using the Finite Difference Time Domain (FDTD) Method. A comparison between the two frequencies on human body tissue will be evaluated.

\section{Numerical Method and Modelling}

A schematic diagram of the multi-layer structure under consideration is shown in Figure 1. It is exposed vertically to electromagnetic waves with frequency equals $900 \mathrm{MHz}$ and $1800 \mathrm{MHz}$ upon the interface distance between the air and the first life tissue is $30 \mathrm{~cm}$ respectively. The electric field is assumed to propagate in the $z$ direction with polarization at the $x$ direction.

The structure has layered media consists of the skin of thickness $d_{1}=0.00073164 \mathrm{~m}$, the fat of thickness $d_{2}=$ 0.02441 , the Muscle of thickness $d_{3}=0.04236$, and the organs (kidney) layer of infinite thickness tissue [8].

The dielectric properties of the layers are donated with the complex permittivity $\varepsilon_{r}^{*}$,

$$
\varepsilon_{r}^{*}=\varepsilon_{r}-j \frac{\sigma}{\omega \varepsilon_{0}}
$$

where $\varepsilon_{r}$ is the real relative part of the permittivity, $\sigma$ is the conductivity and $\omega$ is the radial frequency of the signal. The quantity $\sigma / \omega \varepsilon_{0}$ is called the loss tangent. It describes the looseness of the medium. Since the human tissues are nonmagnetic, it has been assumed that $\mu_{i}=\mu_{0}$, where $i$ stands for $1,2,3$, and 4 which represents the four layers. The free space is assumed for the exterior of the model with wave number $k_{0}=\omega \sqrt{\varepsilon_{0} \mu_{0}}$. And the dielectric properties for each two frequencies are illustrated in Table 1.

\section{Finite Difference Time Domain (FDTD) Method}

The FDTD method solves the curl Maxwell's equations supposing divergence free initial conditions for the field strengths. The method starts with the definition of a generally rectangular mesh (with the choice of the step-sizes $\Delta x, \Delta y$ and $\Delta z$ for the electric field and another staggered (by $\Delta x=2, \Delta y=2$ and $\Delta z=2$ ) grid for the magnetic field in the computational domain. The building blocks of this mesh are the so-called Yee-cells [10].

$$
\begin{aligned}
& \varepsilon \frac{\partial E}{\partial t}=\nabla \times H-J \\
& \frac{\partial H}{\partial t}=-\frac{1}{\mu_{0}} \nabla \times E
\end{aligned}
$$

where $J=\sigma \cdot E$ is the current density.

In 1-D, we consider only $E_{x}$ and $H_{y}$ are not equal to zero and traveling in the $z$-direction. In addition, we assume that the fields do not vary in the $x-y$ plane, i.e.

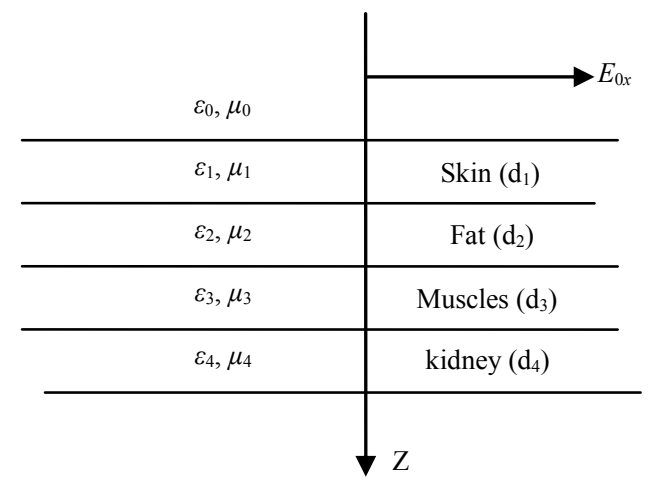

Figure 1. A Electromagnetic handheld cellular phone incident upon the interface between the air and the life tissue.

$\frac{\partial}{\partial x}=0$ and $\frac{\partial}{\partial y}=0$. Then Equations (2) and (3) can be reduced to

$$
\begin{gathered}
\frac{\partial E_{x}(t)}{\partial t}=-\frac{1}{\varepsilon_{0} \varepsilon_{r}} \frac{\partial H_{y}(t)}{\partial z}-\frac{\sigma}{\varepsilon_{0} \varepsilon_{r}} E_{x}(t) \\
\frac{\partial H_{y}(t)}{\partial t}=-\frac{1}{\mu_{0}} \frac{\partial E_{x}(t)}{\partial z}
\end{gathered}
$$

In the FDTD formulation, the central difference approximations for both the temporal and spatial derivatives are obtained at $z=k \Delta z$ and $t=n \Delta t$ [9]

With the above notations the FDTD method computes the numerical solution as follows:

For the first equation [9]:

$$
\begin{aligned}
\frac{E_{x}^{n+1 / 2}(k)-E_{x}^{n-1 / 2}(k)}{\Delta t}= & -\frac{1}{\varepsilon_{0} \varepsilon_{r}} \frac{H_{y}^{n}(k+1 / 2)-H_{y}^{n}(k-1 / 2)}{\Delta z} \\
& -\frac{\sigma}{\varepsilon_{0} \varepsilon_{r}} \frac{E_{x}^{n+1 / 2}(k)+E_{x}^{n-1 / 2}(k)}{2}
\end{aligned}
$$

And for the second equation:

$$
\begin{aligned}
& \frac{H_{y}^{n+1}(k+1 / 2)-H_{y}^{n}(k+1 / 2)}{\Delta t} \\
& =-\frac{1}{\mu_{0}} \frac{E_{x}^{n+1 / 2}(k+1)-E_{x}^{n+1 / 2}(k)}{\Delta z}
\end{aligned}
$$

In the Equations (6)-(7), $n$ is the time index and $k$ is the spatial index, which indexes times $t=n \Delta t$ and positions $z=k \Delta z$, or times $t=\left(n \pm \frac{1}{2}\right) \Delta t$ and positions $z=\left(k \pm \frac{1}{2}\right) \Delta z$. The time index is written as a superscript, and the spatial index is within brackets. Equations (6) and (7) can be rearranged as a pair of "computer update 
Table 1. Dielectric properties for human body at frequency $900 \mathrm{MHz}$ and $1800 \mathrm{MHz}$.

\begin{tabular}{cccccc}
\hline Tissue name & $\begin{array}{c}\text { Conductivity } \boldsymbol{\sigma}[\mathrm{S} / \mathbf{m}] \\
\text { by } \mathbf{9 0 0} \mathbf{~ M H z}\end{array}$ & $\begin{array}{c}\text { Relativepermittivity } \\
\text { by } \mathbf{9 0 0 M H z}\end{array}$ & $\begin{array}{c}\text { Conductivity } \boldsymbol{\sigma} \mathbf{S} / \mathbf{m}] \\
\text { by } \mathbf{1 8 0 0} \mathbf{M H z S} / \mathbf{m}\end{array}$ & $\begin{array}{c}\text { Relative permittivity } \\
\text { by 1800 } \mathbf{M H z}\end{array}$ & Density $\boldsymbol{\rho}\left[\mathbf{k g} / \mathbf{m}^{3}\right]$ \\
\hline Air & 0 & 1 & 0 & 1 & 1.229 \\
Skin & 0.86674 & 41.405 & 1.1847 & 3.8872 & 1100 \\
Fat & 0.051043 & 5.462 & 0.078388 & 5.3494 & 1100 \\
Muscle & 0.94294 & 55.032 & 1.341 & 53.549 & 1040 \\
kidney & 1.3921 & 58.675 & 1.9495 & 54.426 & 1030 \\
\hline
\end{tabular}

equations", which can be repeatedly updated in loop, to obtain the next time values of $E_{x}^{n+1 / 2}(k)$ and $H_{y}^{n+1}(k+1 / 2)$ [9] corresponding the $E_{x}(t+\Delta t / 2, z)$ and $H_{y}(t+\Delta t, z+\Delta z / 2)$. In Equations (4) and (5), $\varepsilon_{0}$ and $\mu_{0}$ differ by several orders of magnitude.

Equations (6) and (7) become

$$
\begin{aligned}
\tilde{E}_{x}^{n+1 / 2}(k) & =\frac{1-\frac{\Delta t \cdot \sigma}{2 \varepsilon_{0} \varepsilon_{r}}}{1+\frac{\Delta t \cdot \sigma}{2 \varepsilon_{0} \varepsilon_{r}}} \tilde{E}_{x}^{n-1 / 2}(k) \\
& -\frac{1 / 2}{\varepsilon_{0}\left(1+\frac{\Delta t \cdot \sigma}{2 \varepsilon_{0} \varepsilon_{r}}\right)}\left[H_{y}^{n}(k+1 / 2)-H_{y}^{n}(k-1 / 2)\right]
\end{aligned}
$$$$
H_{y}^{n+1}(k+1 / 2)=H_{y}^{n}(k+1 / 2)
$$$$
-\frac{1}{\sqrt{\varepsilon_{0} \mu}} \frac{\Delta t}{\Delta z}\left[\tilde{E}_{x}^{n+1 / 2}(k+1)-\tilde{E}_{x}^{n+1 / 2}(k)\right]^{(9)}
$$

Stability and the FDTD method: For stability purposes, we need to choose the cell size $\Delta z$ to allow 10 to 15 points per wave length. We used in all our simulations a time step $\Delta t=\frac{\Delta z}{2 c_{0}}$ where $c_{0}$ is the speed of light in free space. The most important points in FDTD calculations are the stability and numerical dispersion [12].

\section{Results and Discussion}

Simulation of a sinusoidal wave hits a life tissue that has different a dielectric constant according to different frequencies as shown in Table 1. The pulse is generated at the far left side with distance $30 \mathrm{~cm}$ and originates at cell number 2 and propagates to the right. This is illustrated graphically. Notice that the waveform in the medium is maximum absorbed at skin as the first layer, power density and specific absorption rate have been evaluated in life tissue where the power density $\left(\mathrm{W} / \mathrm{m}^{3}\right)$ absorbed in the conductivity $\sigma$ along the layer from the sinusoidal field of the amplitude $E$ is given [8].

$$
P=\frac{E^{2} \sigma}{2}
$$

And specific absorption rate (SAR) in units of $(\mathrm{W} / \mathrm{kg})$ is the most important dosimetric parameter for the evaluation of the exposure hazard at radio and microwaves frequencies $[13,14]$. It is the biological electromagnetic estimation. It is defined as the power dissipation rate normalized by material density [15].

It can be shown that:

$$
S A R=\frac{P}{\rho}
$$

where $\rho_{i}\left(\mathrm{Kg} / \mathrm{m}^{3}\right)$ is the density of each tissue.

The numerical algorithm for Maxwell's curl equations requires that the time increment must have a specific bound relative to the spatial discretization $\Delta t, \Delta x, \Delta y$ and this condition is known as the Courant-Friedrichs-Lewy (CFL) Stability Criterion by

$$
\Delta t \leq \frac{1}{c \sqrt{\frac{1}{\Delta x^{2}}+\frac{1}{\Delta y^{2}}+\frac{1}{\Delta z^{2}}}}
$$

where, $c$ is the speed of light in free space. Simulations were run for mobile phone radiation of the human life tissues at distance $30 \mathrm{~cm}$ which frequency was $0.9 \mathrm{GHz}$ and $1.8 \mathrm{GHz}$ respectively are continuous wave form source.

Figure 2 illustrates the Simulation of the propagation of the electric field of $900 \mathrm{MHz}$ at (a) [8], and $1800 \mathrm{MHz}$ at (b) into life tissue. It can be seen that the maximum peak of incident electromagnetic waves is at cell 32 which represent a skin layer, and it is decrease as the waves penetrate the other tissue. It mean that the tissues are effected and absorption the electromagnetic fields.

The penetration and absorption by $900 \mathrm{MHz}$ is more than $1800 \mathrm{MHz}$ of Power density and Specific absorption rate (SAR) distribution is shown in Figures 3 and 4.

The second peak value in the case of SAR and power is at 30 and 34 time steps respectively. It represents a fat tissue. In Figure 4, the power density was plotting with respect to time steps and the results show a maximum peak is more amplitude than SAR curve peaks. Also the power density at frequency $1.8 \mathrm{GHz}$ is low comparing with the effect of $0.9 \mathrm{GHz}$ frequency. In each curve the muscles tissue start at 58 time steps and kidney at 62 . 


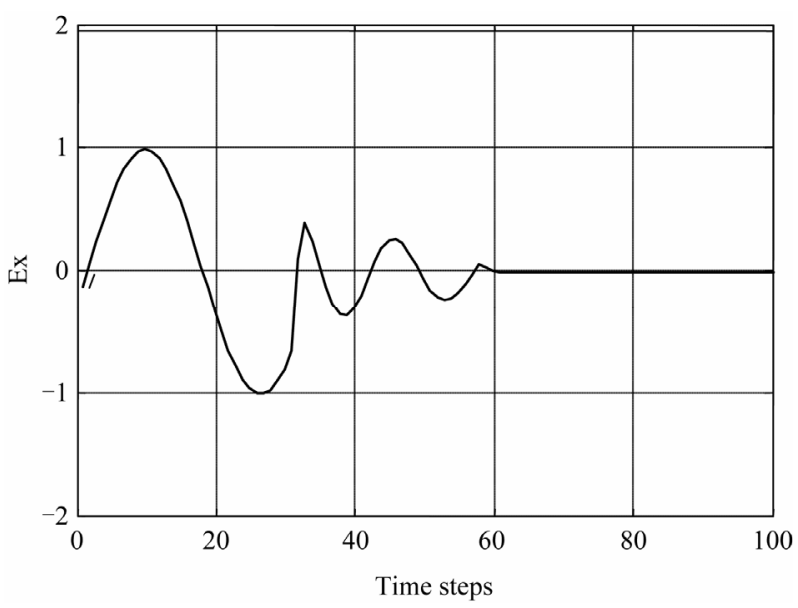

(a)

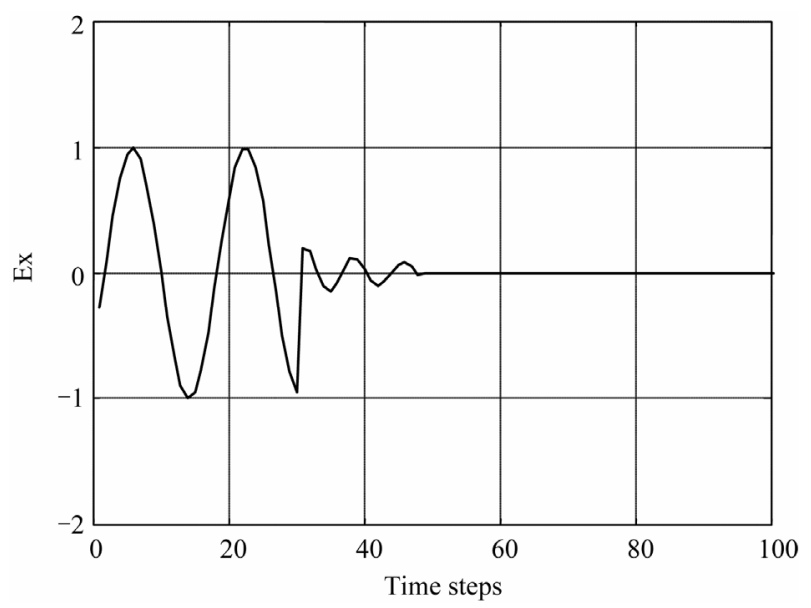

(b)

Figure 2. Comparison the effect of electric field penetration in human body tissue with different frequencies $900 \mathrm{MHz}$ at (a) and $1800 \mathrm{MHz}$ at (b).

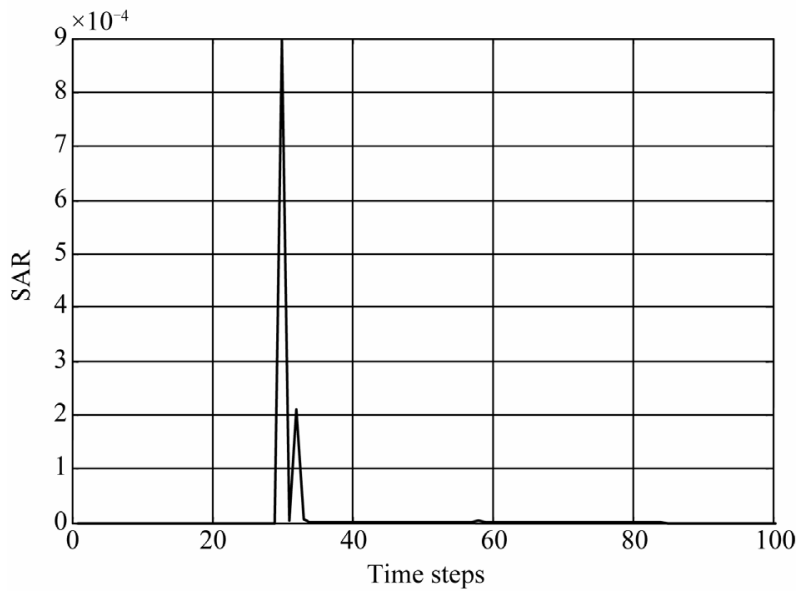

(a)

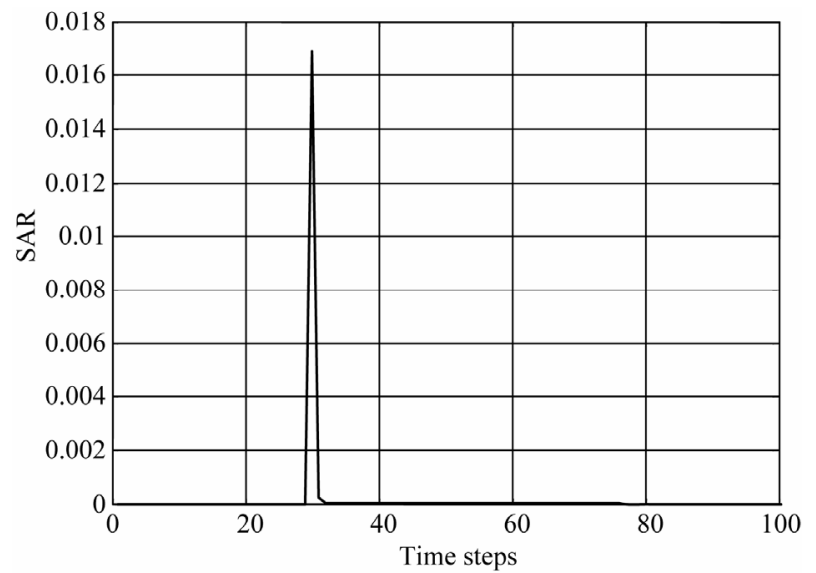

(b)

Figure 3. SAR in life tissues with different frequencies (900 $\mathrm{MHz}$ at (a) and $1800 \mathrm{MHz}$ at (b)).

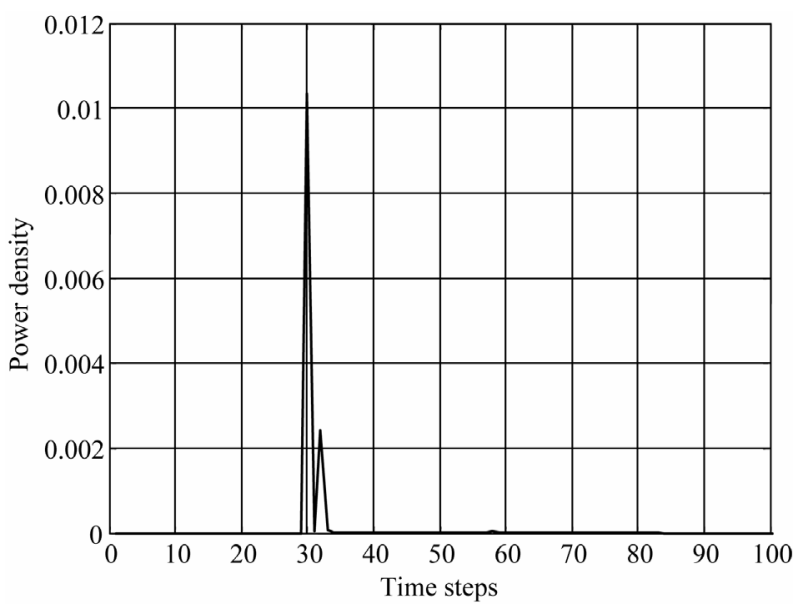

(a)

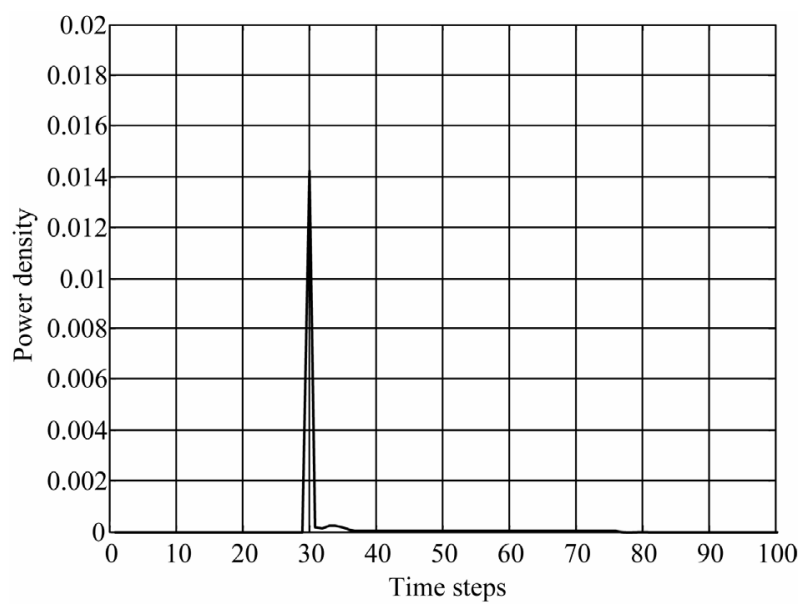

(b)

Figure 4. Tow different frequencies to evaluate Power density in life tissues (900 MHz at (a) and $1800 \mathrm{MHz}$ at (b)). 
This means that there is not any absorption in the later layer instead the electric field at frequency $0.9 \mathrm{MHz}$ in muscles tissue.

\section{Conclusions}

Geometric of human body tissue with four layers has been supposed. The computer equations depend on FDTD method and simulation a sinusoidal wave hitting a lossy medium that has a dielectric constant and conductivity according the frequency which considered has been studied. It is found that the electromagnetic fields penetrates the considered layer and attenuates rapidly till they reach zero at the kidney layer. A comparison of the effect of two frequencies on human body tissue has shown graphically. The results noticed that the effect of frequency $900 \mathrm{MHz}$ by mobile phone Global System Mobile (GSM) is more than the frequency of $1800 \mathrm{MHz}$ in each case as electric field, SAR and power density versus time steps.

Absorbent power and SAR have maximum values at the skin and fat layers. According to the result from the graphs, notice that the effect of frequency $900 \mathrm{MHz}$ by mobile phone Global System Mobile (GSM) is more than the frequency $1800 \mathrm{MHz}$. The dielectric properties for tissue are different according the change in frequency. This model enabled us to make simulations with many different tissues in human body.

\section{REFERENCES}

[1] C. R. Fernández, G. Bulla, A. C. Pedra and A. A. A. de Salles, "Comparison of Electromagnetic Absorption Characteristics in the Head of Adult and a Children for 1800 $\mathrm{MHz}$ Mobile Phones," IEEE Transactions on Microwave Theory and Techniques, Vol. 49, No. 12, 2001, pp. 25392546.

[2] N. Homsup and T. Jariyanorawiss, "FDTD Simulation of a Mobile Phone Operating near a Metal Wall," Journal of Computers, Vol. 4, No. 2, 2009, pp. 168-175. doi.:10.4304/jep.4.2.168-175

[3] R. Luebbers, C. W. Penney and Remcom Inc, "FDTD Comparison of SAR and Temperature Rise in Human
Tissue," IEEE Electromagnetic Compatibility, Vol. 3, No. 1, 2004, pp. 1036-1038.

[4] P. Bernardi, M. Cavagnaro, S. Pisa and E. Piuzzi, "SAR Distribution and Temperature Increase in an Anatomical Model of the Human Eye Exposed to the Field Radiated by the User Antenna in a Wireless LAN," IEEE Transactions on Microwave Theory and Techniques, Vol. 46, No. 6, 1998, pp. 2074-2082. doi:10.1109/22.739285

[5] G. Emili and A. Schiavoni, "Computation of Electromagnetic Field Inside a Tissue at Mobile Communications Frequencies," IEEE Transactions on Microwave Theory and Techniques, Vol. 51, No. 1, 2003, pp. 178-186. doi:10.1109/TMTT.2002.806899

[6] A. Talflove, "Computational Electrodynamics," Artech House, Norwood, 1995.

[7] A. Abdalla and A. Teoh, "A Multi-Layered Model of Human Head Irradiated by Electromagnetic Plane Wave of $100 \mathrm{MHZ}$ - $300 \mathrm{GHZ}$," International Journal of Scientific Research, Vol. 15. No. 2, 2005, pp. 1-7.

[8] H. Elkhozonar, M. Shabat and K. Elwasife, "Modeling the Effect of Electromagnetic Waves Produced by Mobile Phone," AlAzhar Journal of Natural Science, Vol. 12, No. 1, 2010, pp. 81-87.

[9] D. M. Sullivan, "Electromagnetic Simulation Using the FDTD Method," IEEE Microwave Theory and Society, 1999.

[10] M. Witting, S. Burkhardt and SICAN Research \& Development $\mathrm{GmbH}$, "Automatic Generation of Finite Difference Meshes by an Evolutionary Algorithm," IEEE Transactions on Magnetics, Vol. 32, No. 36, 1996, pp. 1338-1340. doi:10.1109/20.497493

[11] IEEE C95.1-1991, "IEEE Standards for Safety Levels Respects to Human Exposure to Radio Frequency Electromagnetic Fields, $3 \mathrm{KHz}$ to $300 \mathrm{GHz}$," Institute of Electrical and Electronics Engineers, Inc., New York, 1992.

[12] U. A. Fill, M. Zank1, N. Petoussi-Henss, M. Siebert and D. Regulla, "Adult Female Voxel Models of Different Stature and Photon Conversion Coefficients for Radiation Protection," Health Physics, Vol. 86, No. 3, 2004, pp 253-272.

[13] J. X. Li. and Z. Q. Niu, "Theory of Biological Electromagnetic," Xidin University Press, Xi'an, 1990. 